\title{
Expanding VLBI in East Asia and AGN Science
}

\section{Kazuhiro Hada ${ }^{* 1,2}$, Bong Won Sohn ${ }^{3}$, Motoki Kino ${ }^{1,4}$, Gabriele Giovannini ${ }^{5}$, and the EAVN AGN Science Working Group}

${ }^{1}$ Mizusawa VLBI Observatory, National Astronomical Observatory of Japan, 2-21-1 Owasa, Mitaka, Tokyo 181-8588, Japan

${ }^{2}$ Department of Astronomical Science, The Graduate University for Advanced Studies (SOKENDAI), 2-21-1 Osawa, Mitaka, Tokyo 181-8588, Japan

E-mail: kazuhiro.hadadnao.ac.jp

${ }^{3}$ Korea Astronomy and Space Science Institute (KASI), 776 Daedeokdae-ro, Yuseong-gu, Daejeon 305-348, Korea

${ }^{4}$ Kogakuin University, Academic Support Center, 2665-1 Nakano, Hachioji, Tokyo 192-0015, Japan

${ }^{5}$ INAF Istituto di Radioastronomia, Via Gobetti 101, 40129 Bologna, Italy

${ }^{6}$ Dipartimento di Fisica e Astronomia, Universitá di Bologna, Via Gobetti 93/2, 40129 Bologna, Italy

The international VLBI collaboration in East Asia is rapidly growing. Besides the successful integration of KVN and VERA (KaVA), now the network is expanding into China. This forms the East Asian VLBI Network (EAVN) and stimulates the activities of joint science promotion among researchers in this area. One of the primary science goals of KaVA/EAVN is to understand the physics of accretion and ejection in active galactic nuclei (AGN) and associated high-energy phenomena. The capability of quasi-full-year operation of KaVA/EAVN is suitable for monitoring the detailed structural evolution of relativistic jets. This allows us to address some major questions about jets such as the acceleration mechanisms, connection to gamma-ray flares and magneticfield properties. Some of key AGN programs are project-led and promoted by the EAVN AGN Science Working Group. This includes a massive monitoring program of SgrA* and M87 near in time to the Event Horizon Telescope observations, EAVN+Italy global VLBI ( "EATING" VLBI) observations of powerful jets, as well as monitoring of other individual sources. Here we overview the ongoing expansion of EAVN and related AGN observing programs.

14th European VLBI Network Symposium \& Users Meeting (EVN 2018)

8-11 October 2018

Granada, Spain

\footnotetext{
${ }^{*}$ Speaker.
} 


\section{Introduction}

Active galactic nuclei (AGN) are believed to be powered by the accretion onto the central supermassive black holes (SMBH). Approximately ten percent of them are known to be "radioloud", exhibiting powerful relativistic jets. Understanding the formation and emission processes of the relativistic jets in AGN is a longstanding goal in high-energy astrophysics. The inner jet regions at parsec to subparsec scales are particularly relevant to the acceleration, collimation, and production of high-energy $\gamma$-ray emission in the jets. High-resolution VLBI plays a key role in addressing these questions since it can directly resolve, image, and monitor the relevant scales.

Recently, observations of AGN jets with VLBI facilities in East Asia are growing rapidly [प, ఒ, [1, 田, [1, 田, 四]. In particular, the start of the KVN and VERA Array (KaVA) in 2014 was a milestone for the VLBI community in East Asia, since it yielded in this region the first VLBI array with nice imaging performance [ [8]]. In addition, KaVA operates for a quasi-full year. This opens a new opportunity for detailed high-resolution monitoring studies of AGN jets along with other existing VLBI facilities such as the VLBA. An increasing number of sources including nearby radio galaxies, $\gamma$ -

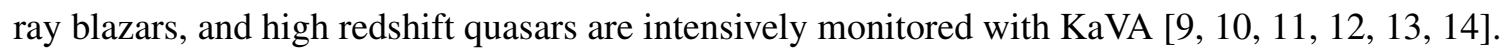
However, KaVA is just the first step towards the larger East Asian collaboration including China and other nearby countries. Here we introduce the recent growing VLBI collaboration in East Asia and some ongoing research programs on AGN based on the East Asian VLBI Network.

\section{The East Asian VLBI Network}

East Asia is one of the particular areas in the world where a number of radio telescopes are densely distributed. In China, Korea and Japan, there are their own VLBI networks such as CVN, KVN, JVN and VERA. The East Asian VLBI Network (EAVN, see also Figure $\amalg$ ) is a collaborative effort among these countries aiming to form a large international VLBI network by combining these

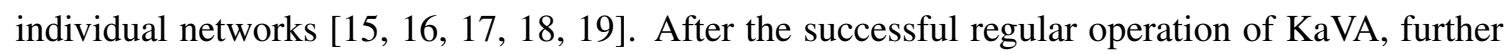
efforts have been made to expand the network into China as well as other stations in Korea/Japan.

Following a commissioning phase lasting a few years, from late-2018, EAVN has started its regular operation as an extension of KaVA, by including Tianma $65 \mathrm{~m}$, Nanshan $26 \mathrm{~m}$ and Nobeyama $45 \mathrm{~m}$ telescopes. The EAVN open-use capability supported as of 2018 November is summarized

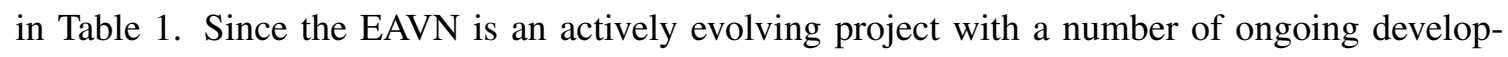
ments (e.g., observing mode, frequency, recording rate, polarization, the number of participating telescopes), the performance summarized in the table will be continuously updated with time. Nevertheless, thanks to the addition of the large dishes to KaVA, the overall array sensitivity is already significantly increased (by a factor of $\sim 4$ compared to KaVA). The angular resolution at $22 \mathrm{GHz}$ is also $\sim 2.5$ times sharper than KaVA due to the addition Nanshan/Urumqi.

\section{AGN science with EAVN}

Various scientific programs are ongoing with EAVN through the open-use time that any individuals or research groups can propose. Apart from these, some AGN key science programs with EAVN are promoted by the EAVN AGN Science Working Group, which is a joint team composed 


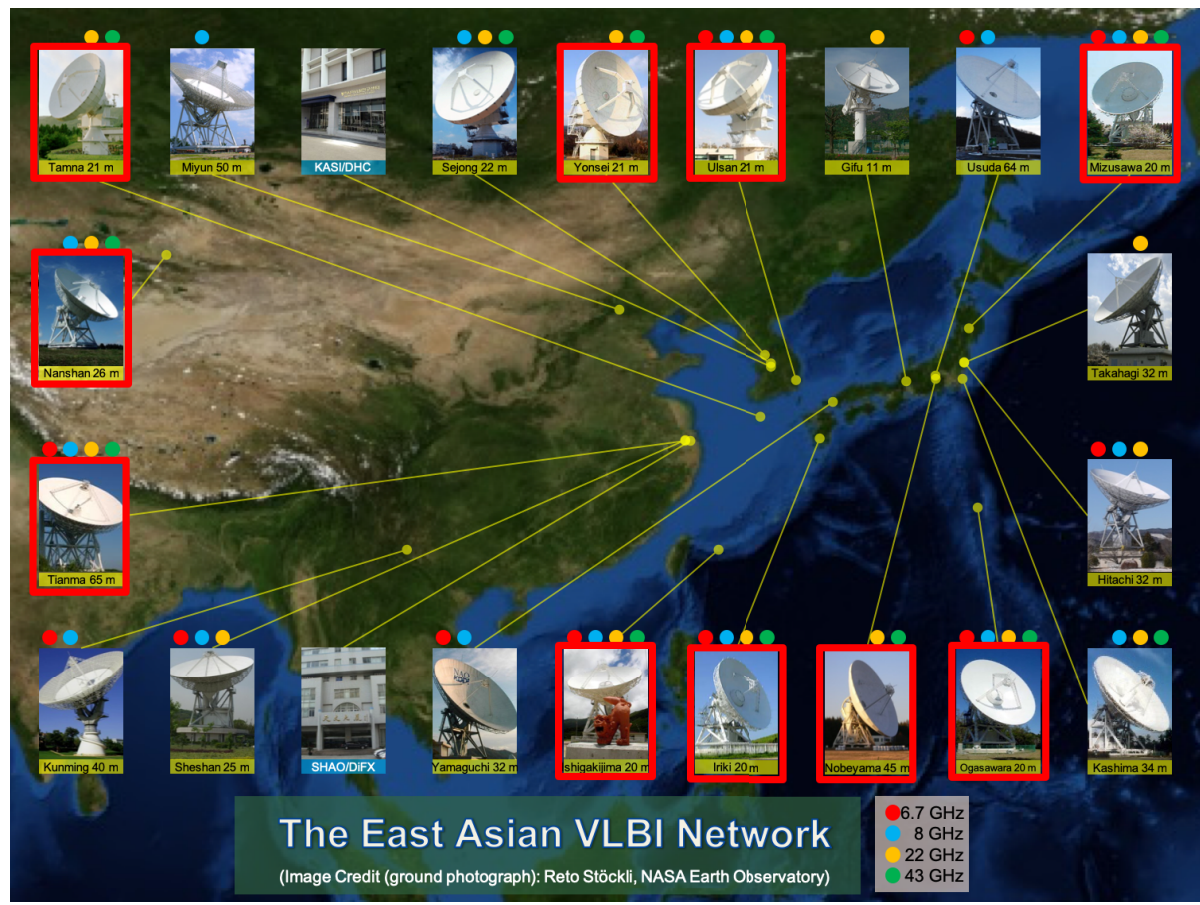

Figure 1: The East Asian VLBI Network. Shown here are the stations where the frequency bands between 6.7 $\mathrm{GHz}$ and $43 \mathrm{GHz}$ are equipped. The stations highlighted by red are the ones that are available for the current EAVN open-use.

Table 1: Performance of EAVN supported for the current open-use observations.

\begin{tabular}{ll}
\hline \hline Available stations $(22 \mathrm{GHz})$ & KaVA-7, Tianma65, Nobeyama45, Nanshan26 \\
Available stations $(43 \mathrm{GHz})$ & KaVA-7, Tianma65, Nobeyama45 \\
Recording mode & $1 \mathrm{Gbps}, \Delta v=256 \mathrm{MHz}$, single polarization \\
Maximum fringe spacing & $0.53 \mathrm{mas}(22 \mathrm{GHz}), 0.64 \mathrm{mas}(43 \mathrm{GHz})$ \\
Baseline sensitivity $(1 \sigma, \tau=60 \mathrm{sec})$ & $1.1 \mathrm{mJy}(22 \mathrm{GHz}), 1.7 \mathrm{mJy}(43 \mathrm{GHz})$ for TM65-NRO45 \\
Array sensitivity $(1 \sigma, \tau=60 \mathrm{~min})$ & $35 \mu \mathrm{Jy} / \mathrm{beam}(22 \mathrm{GHz}), 65 \mu \mathrm{Jy} / \mathrm{beam}(43 \mathrm{GHz})$ \\
Correlator & Daejeon hardware correlator \\
\hline
\end{tabular}

by members from various institutes in East Asia. In particular, we are promoting intensive EAVN monitoring observations of M87 and SgrA*. These two sources are known to be the primary targets for the Event Horizon Telescopes (EHT), and provide the best opportunity to investigate the physics of accretion and jet formation near the central black holes. Our program originally started in 2014 as a large program of KaVA, but from 2017 this program was expanded by including Chinese stations. M87 is observed every 2-3 weeks at 22 and $43 \mathrm{GHz}$, while SgrA is monthly monitored at $43 \mathrm{GHz}$. Especially in the spring 2017/2018 where M87/SgrA* were observed with EHT, we intensified our EAVN monitoring of these sources to better constrain their structural evolution. Figure $\square$ is an example EAVN image of M87 that was taken in March 2017. The EAVN image 

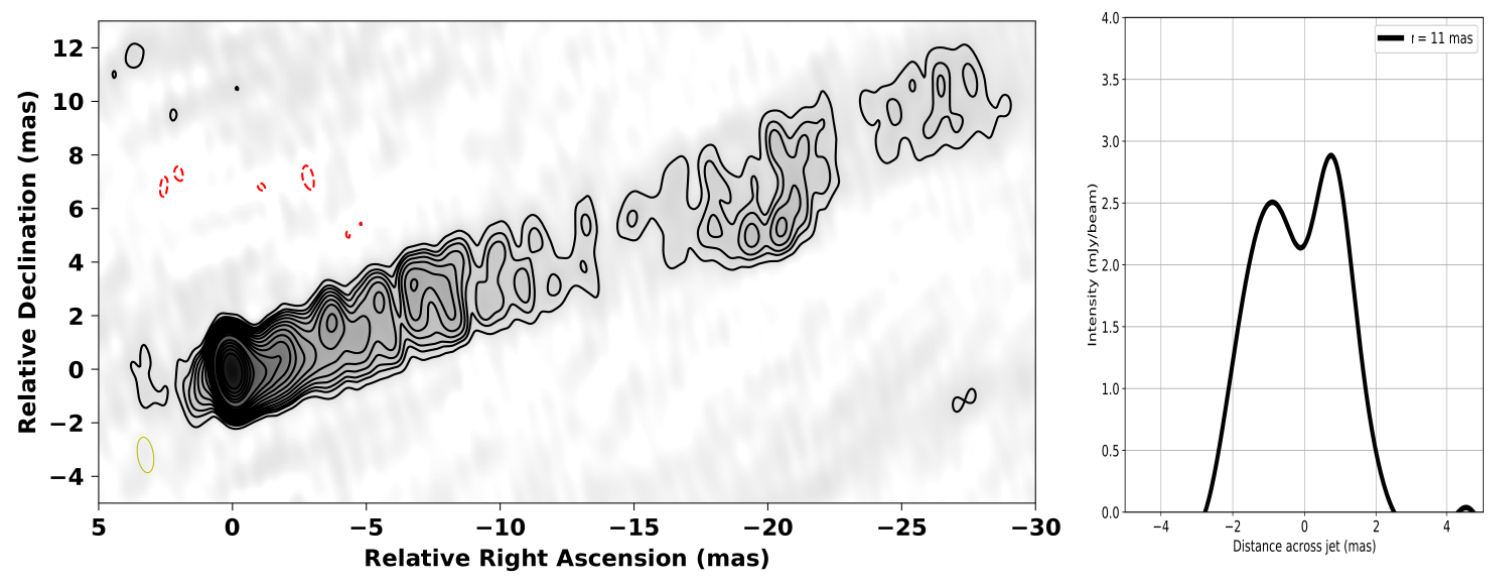

Figure 2: (Left) EAVN $22 \mathrm{GHz}$ image of M87. The observation was made on March 18th 2017 with the stations of KaVA, Tianma $65 \mathrm{~m}$, Nanshan $26 \mathrm{~m}$ and Hitachi $32 \mathrm{~m}$. (Right) Transverse jet slice profile of M87 at 11 mas from the core. The well-known limb-brightened jet pattern is clearly resolved in the EAVN image.

clearly resolved the limb-brightened profile of this jet. More detailed analyses of these massive data, such as jet collimation profile and proper motion measurements, are in progress.

Beyond EAVN, we are also expanding our array by collaborating with the Italian VLBI Network. By combing EAVN and Italy, we can obtain $\sim 10000 \mathrm{~km}$ baselines, which realizes a nearly global VLBI (East-Asia-To-Italy-Nearly-Global VLBI, or EATING VLBI). Bright jet sources in the northern hemisphere such as M87 and 3C84 are primary targets of this array (Figure B). For M87, between March and May 2018, we have succeeded in coordinating frequent ( biweekly) EATING monitoring observations, which is generally very challenging to realize for a global VLBI. We are still working on these data, but our preliminary analysis resolves the jet base of M87 at scales of $\sim 18 R_{\mathrm{s}}$, which is comparable to the scales of the EHT. This indicates that EATING VLBI would be useful as a guide to properly interpret the contemporaneous EHT images. As for 3C84, this is a very good target for EATING VLBI because it is a high declination source and there is sufficient common sky between East Asia and Italy (Figure B]). Other famous high declination sources such as BL Lac, Mrk421, Mrk501, OJ287, Cygnus A, 1H 0323+342 and 0716+714 are also suitable targets for this array. Future EATING monitoring of these sources at $22 / 43 \mathrm{GHz}$ will uniquely determine the structural evolution of these jets at scales of $\sim 70 \mu$ as. Our EATING VLBI activities might also be expanded by including other EVN stations or existing VLBI networks such as VLBA or LBA, which would realize a genuine global VLBI array.

\section{Future plan}

EAVN is an ongoing, continuously expanding project. More stations in East Asia and also in Southeast Asia (e.g., Thailand National Radio Telescope) are planned to join the network in the near future. For studies of AGN jets, an important function is the dual-polarization capability. While KVN and CVN already support dual-polarization, VERA is currently on the way of upgrading this function. We plan to complete the dual-polarization upgrade at all the four VERA stations 

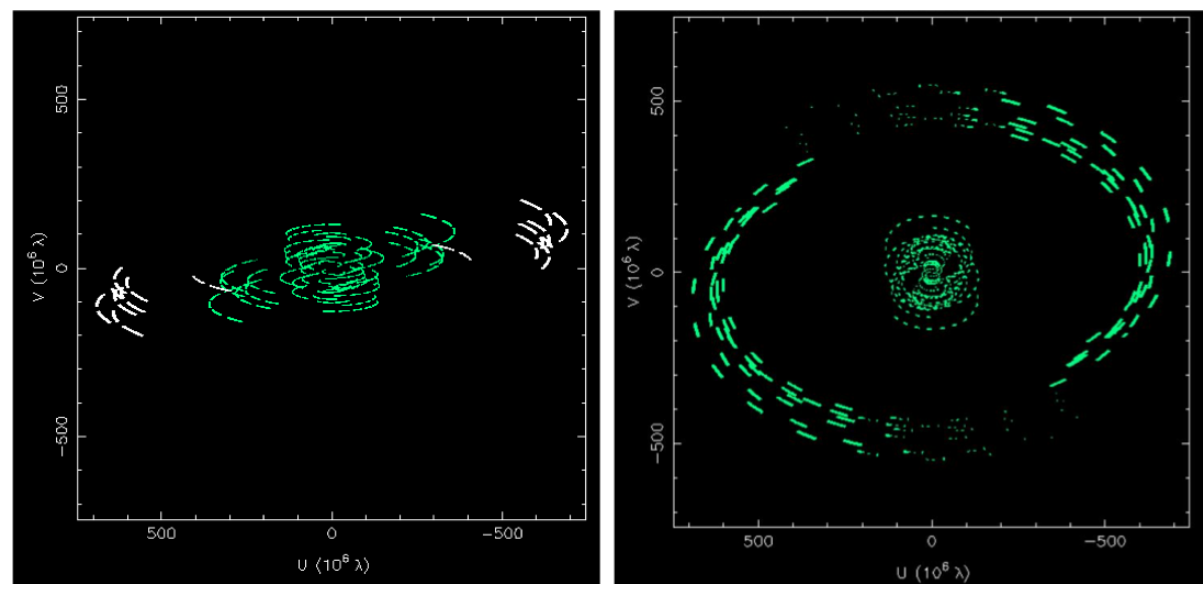

Figure 3: Examples of uv-coverage from previous EATING VLBI experiments at $22 \mathrm{GHz}$. (Right) M87 observed by KaVA, Tianma, Nanshan, Hitachi and Medicina. (Left) 3C84 observed by KaVA, Medicina and Noto.

during 2019. Another unique function of EAVN is the K/Q simultaneous observing capability. This is originally developed by $\mathrm{KVN}$, but the compatible system has been installed at VERA, and more other stations plan to install this system. This capability will allows us to determine simultaneous spectra of jets and also help detect fringes at the higher frequency. We are also planning to promote EAVN at low frequencies such as L band $\mathrm{C}$ band, which can be compatible with the FAST $500 \mathrm{~m}$ telescope. Low-frequency EAVN observations with FAST will be very complementary to the Square Kilometer Array, and may allow us to probe the evolution of AGN from the present to the early Universe at the highest resolution.

Acknowledgments. KVN is a facility operated at by the Korea Astronomy and Space Science Institute. VERA is a facility operated at National Astronomical Observatory of Japan in collaboration with associated universities in Japan. This work was supported by the Sumitomo Foundation Fiscal 2018 Grant for Basic Science Research Projects (grant number 170201).

\section{References}

[1] Nagai, H., Kino, M., Niinuma, K., et al. 2013, PASJ, 65, 24

[2] Hada, K., Giroletti, M., Kino, M., et al. 2014, ApJ, 788, 165

[3] Lee, S.-S., Wajima, K., Algaba, J.-C., et al. 2016, ApJS, 227, 8

[4] Kim, J.-Y., Trippe, S., Sohn, B.W., et al. 2015, JKAS, 48, 285

[5] Park, J., Kam, M., Trippe, S., et al. 2018, ApJ, 860, 112

[6] Hiura, K., Nagai, H., Kino, M., et al. 2018, PASJ, 70, 83

[7] Fujinaga, K., Niinuma, K., Kimura, A., et al. 2018, PASJ, 68, 70

[8] Niinuma, K., Lee, S.-S., Kino, M., et al. 2014, PASJ, 66, 103

[9] Oh, J., Trippe, S., Kang, S., et al. 2015, JKAS, 48, 299 
[10] An, T., Cui, Y.-Z., Paragi, Z., et al. 2016, PASJ, 68, 77

[11] Zhang, Y., An, T., Frey, S., et al. 2017, MNRAS, 468, 69

[12] Zhao, G.-Y., Kino, M., Cho, I., et al. 2017, IAUS, 322, 56

[13] Hada, K., Park, J., Kino, M., et al. 2017, PASJ, 69, 71

[14] Kino, M., Wajima, K., Kawakatsu, N., et al. 2018, ApJ, 864, 118

[15] Wajima, K., Hagiwara, Y., An, T., et al. 2016, ASPC, 502, 81

[16] An, T., Sohn, B., \& Imai, H. 2018, Nature Astronomy, 2, 118

[17] Fujisawa, K., Sugiyama, K., Motogi, K., et al. 2014, PASJ, 66, 31

[18] Sugiyama, K., Fujisawa, K., Hachisuka, K., et al. 2016, PASJ, 68, 72

[19] Cho, I., Jung, T., Zhao, G.-Y., et al. 2017, PASJ, 69, 87 